\title{
Marina Ratner
}

\section{S. G. Dani}

This article has been reproduced with permission from Bhāvanā, October 2017, Vol. 1, Issue 4. See bhavana.org . in/marina-ratner-1938-2017.

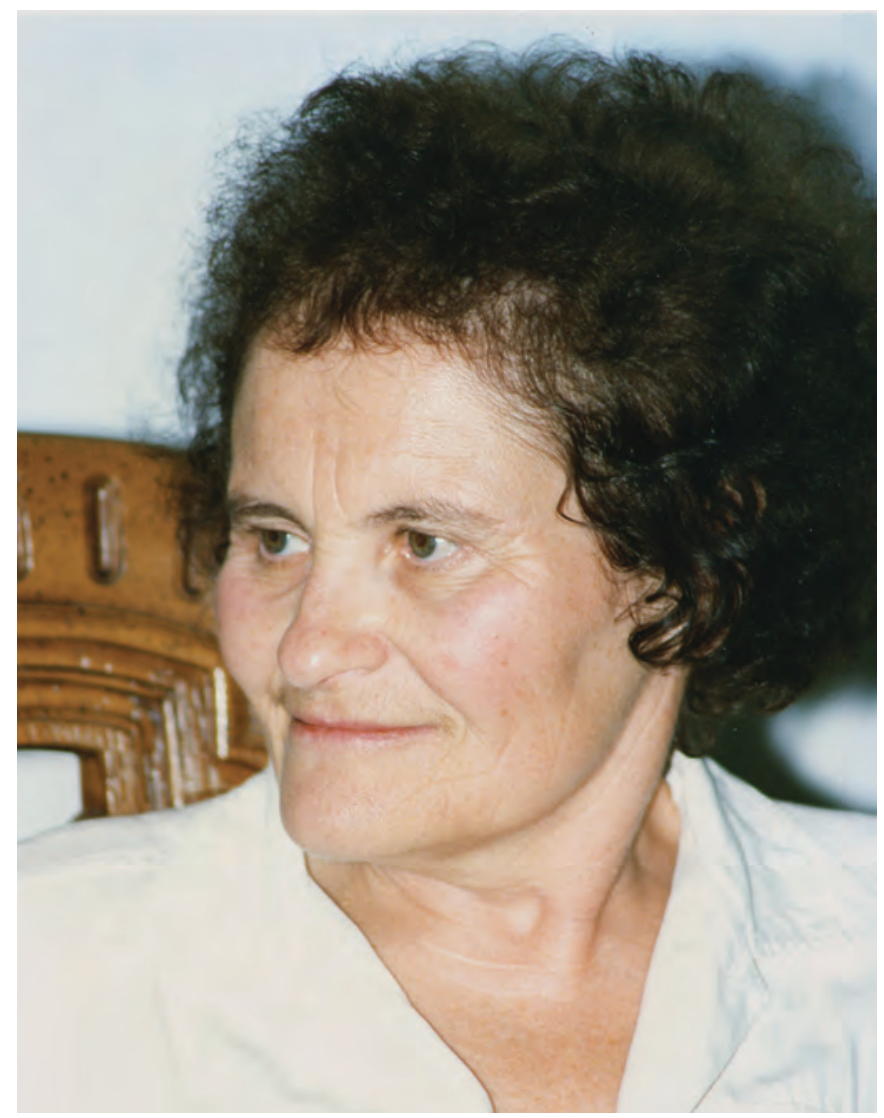

S. G. Dani was affiliated with the Tata Institute of Fundamental Research (TIFR), Mumbai for over four decades, until mandatory retirement in 2012. Subsequently, he was associated with IIT Bombay and, more recently, is with the UM-DAE Centre for Excellence in Basic Sciences, Mumbai, a collaborative endeavor of the University of Mumbai and the Department of Atomic Emergy of the Government of India. His email address is shrigodani@gmai1. com.

For permission to reprint this article, please contact: reprint -permission@ams.org.

DOI: http://dx.doi.org/10.1090/noti1835
Marina Evseevna Ratner, renowned for her work in dynamics, passed away on July 7, 2017, at her home at El Cerrito, California, USA, at the age of 78 . Her profound contributions, establishing the Raghunathan conjecture and its variants, from the 1990s when she was in her early fifties, have become a milestone in homogeneous dynamics and have had an impact on the study of a broad range of areas of mathematics, including dynamics, diophantine approximation, ergodic theory, geometry, and Lie group theory.

Marina was born in Moscow on October 30, 1938 to scientist parents, her father a plant physiologist and mother a chemist. As a Jewish family they had a difficult time in Russia at that time. In particular, her own mother lost her job for having corresponded with none other than her mother who was in Israel, which was considered an enemy state. Marina was educated in Moscow and fell in love with mathematics when she was in the fifth grade; "mathematics came naturally to me and I felt unmatched satisfaction solving difficult problems" she was to aver later. ${ }^{1}$ After completing school she gained admission to the Moscow State University, which, with the dawning of the Khrushchev era, had begun to accept Jewish students on an equal footing.

After graduating from the University in 1961, Ratner worked for four years as an assistant in the Applied Statistics Group of A. N. Kolmogorov, the celebrated Russian mathematician who laid the foundations of measure-theoretic probability theory and had a great influence on her during her undergraduate years. Kolmogorov had an intensive training program for talented high school students with which Marina was actively involved. It is also during these years that she gave birth to a daughter from a short-lived marriage.

\footnotetext{
${ }^{1}$ M. Cook, Marina Ratner, in: Mathematicians: An Outer View of the Inner World, Princeton University Press, 2009, pp. 90-91.
} 
In 1965 Marina took up research under the supervision of Ya. G. Sinai, a former student of Kolmogorov, renowned, in particular, for his role jointly with his advisor in the development of the very influential "entropy" invariant in ergodic theory around $1960 .{ }^{2}$ In the context of how the theory was developing then, in Russia, the geodesic flows associated with surfaces of negative curvature had emerged as crucial examples for study from an ergodic-theoretic point of view, and Ratner also wrote her thesis on this topic. ${ }^{3}$ Apart from the examples themselves, a general class of systems known as Anosov flows, named after D. $\mathrm{V}$. Anosov who introduced and proved some deep results about them, were of interest, and Ratner worked on the asymptotic statistical properties of these flows as well. For the work she received the equivalent of the $\mathrm{PhD}$ degree in 1969 from Moscow State University.

After receiving the degree Ratner was employed as an assistant at the High Technical Engineering School in Moscow. In 1970 the government of USSR was led, in the face of international pressure, to increase substantially the emigration quotas, sparking an exodus of Russian Jews to Israel, of which earlier there had just been a trickle. Notwithstanding the relaxation in the policy, the government and the bureaucracy in general were highly resistant to emigration and treated those desirous of migrating with utmost severity in various ways. Thus, when Ratner applied for a visa that year (1970) to emigrate to Israel, she was dismissed from the job at the Engineering School.

\footnotetext{
${ }^{2} \mathrm{~A}$ dynamical system in the present context means a one-parameter group of transformations of a space (sometimes called the phase space), with the parameter representing time (which could be continuous or discrete); the theory focuses on the long-term behavior of the trajectories of points under application of the transformations, namely, as the time parameter tends to infinity. In ergodic theory the phase space is further considered equipped with a measure of unit mass, namely, a probability is associated for points to belong to various subsets; the system is said to be measure-preserving if the probability remains unchanged when any of the transformations under consideration is applied to a point. In these instances one often focuses on trajectories of "generic points" in terms of the measure or equivalently in statistical terms with respect to the initial point. The Kolmogorov-Sinai entropy is a nonnegative number associated with each measure-preserving system, and when the entropy of two systems is different, their long-term behavior is different. The invariant thus enabled distinguishing dynamical systems on a much finer scale than was possible before.

${ }^{3}$ The "geodesic flow" consists of starting with a given point on the surface (or manifold in general) and a direction from that point and moving on, along the distance-minimizing paths corresponding to the geometry of the surface, for the desired amount of time, and noting the point of arrival and the direction of movement at that point; thus, the phase space in this case is formed of pairs consisting of a point of the surface and a direction at that point, and the above procedure describes how the transformation is defined. On the usual sphere the trajectories (paths) of such a flow would follow the great circle, and return to the original point after a fixed amount of time. However, when the surface has negative curvature, the trajectories move away from each other substantially, exponentially in time, and when the surface is compact, "almost all" of them (statistically) tend to fill up the whole space with the passage of more and more time.
}

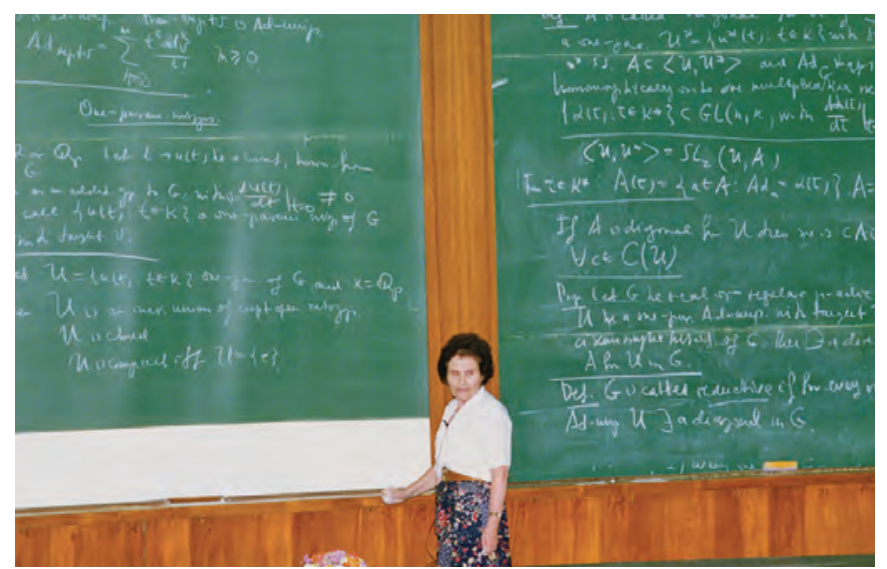

Marina Ratner giving a talk at the International Colloquium at TIFR, 1996.

Ratner landed in Israel, with her daughter, in 1971 and served as a lecturer until 1974 at the Hebrew University of Jerusalem and then at the Pre-academic School of the Hebrew University for another year. During this period Ratner continued her work on the geodesic flows, and also their generalizations in higher dimensions, and established in particular a property manifesting complete randomness of behavior of the trajectories of the Anosov flows, known as the Bernoulli property, that was much sought after in various systems.

In the West the study of flows analogous to Anosov flows, called "Axiom A" flows, was introduced by Stephen Smale, then at the University of California (UC), Berkeley. Apart from a certain generality of setting, this study involves separating the role of the measure and understanding the dynamics in terms of the construction of special kinds of partitions of the space, known as Markov partitions. Profound work was done in this direction by one of Smales's students, Robert E. Bowen, known commonly by his adopted name "Rufus" Bowen; the work led Bowen to the construction of invariant measures inherently associated to the systems in more general settings, now known as Bowen measures. Bowen completed his doctorate in 1970 and joined the Berkeley faculty in the same year. Not surprisingly, Bowen was interested in the work of Marina Ratner, and their correspondence during her Jerusalem years culminated in Ratner getting an invitation from UC Berkeley, which she joined in 1975 as acting assistant professor.

Another class of flows, called horocycle flows, are seen to have become a major love for Marina after moving to Berkeley. The geodesic flow associated with a surface of constant negative curvature has two natural companion flows, called the contracting horocycle flow and the expanding horocycle flow; they are actually twins, interchangeable through time reversal of directions at each point, so one may simply talk of the horocycle flow. Passing through each point of the phase space (consisting of a point of the surface together 
with a direction at the point), there is a uniquely defined curve such that if we pick two points on any one of these curves and consider their trajectories under the geodesic flow we find them getting closer and closer with the passage of time, with the distance between the corresponding points of the trajectories tending to zero. Moreover, there is a natural parametrization on these curves with respect to which they can be thought of as the trajectories of a measure-preserving flow, and that is the (contracting) horocycle flow associated with the surface; the expanding horocycle flow arises similarly from consideration of trajectories of the geodesic flow in the reverse direction. These flows have historically proved to be very useful in studying the properties of the geodesic flows. While in the nature of things the horocycle flow would seem just a sidekick of the geometrically majestic geodesic flow, in the theory of dynamical systems the former has acquired a stature of its own, on account of some of its unique properties.

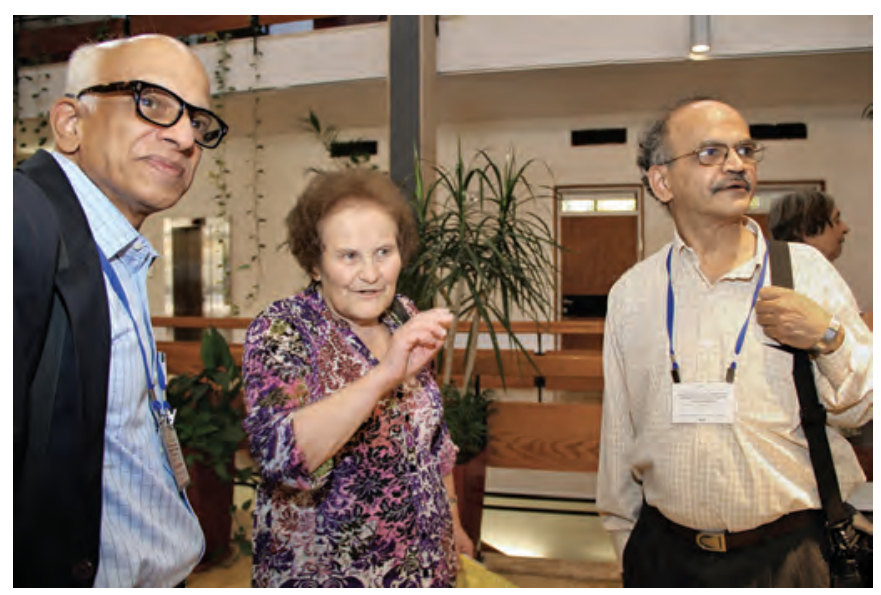

Marina Ratner with M. S. Raghunathan and S. G. Dani at the conference held in her honor at the Hebrew University of Jerusalem in October 2013.

In two papers published in 1978 and 1979 Ratner showed that the horocycle flows are "loosely Bernoulli" while their Cartesian squares are not "loosely Bernoulli"; the loose Bernoullicity property was introduced by J. Feldman, a colleague at Berkeley, and concerns the flow being similar to the standard winding line flows on the torus along lines with irrational slopes, if one allows the time parameter associated with the trajectories to be modified suitably. The fact, as established by Ratner, that the Cartesian square is not loosely Bernoulli for the horocycle flows is rather curious and was the first such instance to be found.

The early 1980s saw a major breakthrough in the understanding of the horocycle flows associated with compact surfaces, of constant negative curvature, at the hands of Ratner. A major question involved was the following: given two such surfaces whether the horocycle flows associated with them being isomorphic to each other as measure-pre- serving flows would imply that the surfaces themselves are geometrically indistinguishable. The answer to the corresponding question in the case of the geodesic flows is a definitive no since in fact the flows being Bernoullian means (by a well-known result of D. S. Ornstein) that any two of them are isomorphic to each other (up to a rescaling of the time parameter), irrespective of the specific geometries of the underlying surfaces. Ratner proved that on the other hand the horocycle flows corresponding to two distinct compact surfaces of constant negative curvature would never be isomorphic. This kind of phenomenon is referred to as rigidity. She also exhibited various variations of the rigidity property of the horocycle flows, through a series of papers, describing their factors, joinings, etc. (joining is a technical construction that enables comparing two systems with regard to the nature of their dynamics). Two of the three papers in this respect appeared, in 1982 and 1983, in the Annals of Mathematics. Apart from the immediate outcomes, which were striking in themselves, the work has germs of the ideas involved in the later celebrated work on the Raghunathan conjecture.

Let me now come to the Raghunathan conjecture, resolution of which was the major feat of Marina's work. Genesis of the conjecture is intricately connected with my student years at the Tata Institute of Fundamental Research, and it would be worthwhile to recall some details in that regard. I did my doctoral work in the early 1970s under the supervision of M. S. Raghunathan on flows on homogeneous spaces. ${ }^{4}$ The thesis dealt primarily with the Kolmogorov property, which is a statistical property concerning a strong form of mixing, with no direct bearing on the behavior of individual orbits. However, in a paper written shortly after completing the thesis paper (before the award of the degree, in fact) I proved that all the orbits of actions of a class of flows, more specifically horospherical flows, are dense in the space. Around that time Jyotsna Dani (my wife) who was working under the supervision of S. Raghavan, at TIFR, had proved that for any vector whose coordinates are nonzero and not rational multiples of each other, the

\footnotetext{
${ }^{4}$ For an idea of homogeneous spaces and dynamics on them let us consider a Euclidean space and agree to identify two given vectors of the space if their difference has integer coordinates, namely, we view the vectors modulo the lattice of vectors with integral coordinates; geometrically in effect we are considering a torus, and translations by vectors on any particular line define a translation flow on the torus. Similarly, when the elements of various matrix groups, more generally Lie groups, are considered modulo elements of large enough discrete subgroups (called lattices) we get what are called homogeneous spaces with a natural finite measure on them, and matrix multiplication by elements from a one-parameter subgroup of the ambient group, considered modulo the lattice, defines a flow on the homogeneous space. In the particular case when the group involved is the "modular group," the group of $2 \times 2$ matrices with real entries and determinant 1 , the flows arising in this way (other than those which are periodic) in fact correspond to the geodesic and horocycle flows associated with various surfaces with constant negative curvature, via certain natural identification of the phase space of the flow with a homogeneous space of the modular group.
} 


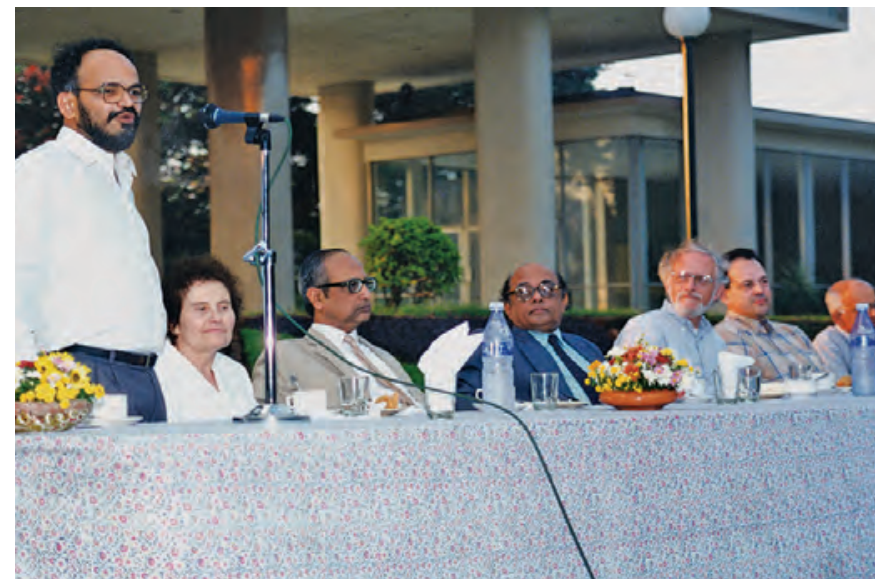

Marina Ratner at the inaugural function of the

International Colloquium on Lie Groups and ErgodicTheory at theTata Institute of Fundamental Research, Mumbai, 1996. The others on the dais, from left to right are, S. G. Dani, Virendra Singh, R. Chidambaram, Hillel Furstenberg, Anatole Katok, and M. S. Raghunathan.

orbit under the action of the group of integral unimodular (determinant 1) matrices on the corresponding Euclidean space, is dense in the Euclidean space. At some point in time around 1975, which had these events in the background, when I was talking to Raghunathan about possible problems to pursue, he casually suggested a statement on the behavior of what are called unipotent flows ${ }^{5}$ and quite nonchalantly added "call it my conjecture and prove it." He pointed out that proving it would in particular settle the conjecture of Oppenheim on density of values of indefinite forms at integral points, ${ }^{6}$ which was one of the hallowed problems at that time in the Tata Institute precincts.

That statement of Raghunathan-the Raghunathan conjecture-first recorded in print in my Inventiones Mathematicae (1981) paper, is that the closure of any orbit of a unipotent one-parameter subgroup acting on a homogeneous space of finite volume is the orbit of a (possibly larger) subgroup of the ambient Lie group; in particular this means that each of these closures of orbits

\footnotetext{
${ }^{5}$ A flow on a homogeneous space of a matrix group as in the previous footnote is said to be unipotent when the one-parameter group involved consists of unipotent matrices, namely, matrices that have no eigenvalue, even in complex numbers, other than 1; for a general Lie group there is a variation of this involved. In the case of the modular group there are precisely the horocycle flows associated with surfaces of constant negative curvature and finite area.

${ }^{6}$ The conjecture originating from a paper of Alexander Oppenheim from 1929 predicted that for any nondegenerate indefinite quadratic form in at least three variables, which is not a multiple of a form with rational coefficients, the set of its values at integer tuples is dense in real numbers. It had been worked on by several notable number theorists, and by the $1980 \mathrm{~s}$ many partial results were known, confirming the conjecture under various restrictions, but a general solution had eluded the efforts.
}

is a geometrically nice object-this is a remarkable thing to happen for a dynamical system, the crucial point being that the statement is being made for every orbit and not only the generic ones. ${ }^{7}$ In that paper I proposed another conjecture, as a step toward proving the Raghunathan conjecture, relating to measures that are invariant under these flows, namely, that the ergodic ${ }^{8}$ ones from among them in fact arise as measures invariant under the action the larger subgroups as above and are supported on a single orbit of the subgroup; a weak result was proved in the paper in that direction, partially vindicating the conjecture. Ratner proved the latter conjecture, which she referred to as "Raghunathan's measure conjecture," and in a separate paper deduced the original topological version. ${ }^{9}$

The Oppenheim conjecture itself, which had inspired the Raghunathan conjecture, was settled by G. A. Margulis in 1986 by proving a much weaker statement than the Raghunathan conjecture but in a similar spirit. Not surprisingly, proving the full conjecture led to a much broader perspective in the study of values of quadratic forms at points with integer coordinates, and many other applications, some quite immediately and many more over the years. There have been numerous results since then making use of Ratner's theorems in crucial ways, in a variety of contexts, and there is no doubt that it will serve as a mainstay for a good deal of mathematics in the coming decades.

The proofs are long and intricate and involve various ancilliary results. However, there is a beautiful key idea that concerns observing and adopting a property of the unipotent flows, which it may be worthwhile to recall. It may be informally stated as the following: if you find two trajectories of the flow having stayed quite close for reasonably long, then you can expect them to stay fairly close for substantially longer. This property of the unipotent flows, now called the Ratner property, has since acquired significance as a dynamical phenomenon.

As to be expected, Ratner gained considerable professional recognition. While her initial appointment at Berkeley had been a source of some controversy in the Department, her subsequent rise in the ranks seems to have

\footnotetext{
${ }^{7}$ For a one-parameter flow the orbit of a point is the set of all the points that can be reached by application of one of the transformations from the flow (including those corresponding to the negative value of the time parameter); similar terminology applies also to a more general group of transformations, in place of the one-parameter flows. The closure of the orbit means all the points that can be approximated by points on the orbit. In a typical dynam$i c a l$ system, even when the closures of almost all orbits are the whole space, for others, the exceptional ones, the closures can be very crazy. For instance, for the geodesic flows as in the above discussion there are orbit closures whose intersection with some curves transversal to the flow consists of a mess of uncountably many individual points disconnected from each other.

${ }^{8}$ Those that cannot be expressed nontrivially as a sum of two invariant measures.

${ }^{9}$ During the interim there were various partial results proved in that direction, but we shall not concern ourselves with it here.
} 
been smooth-sailing. She was elected in 1992 to the American Academy of Arts and Sciences, and in 1993 she was awarded the Ostrowski Prize. ${ }^{10}$ In 1994 she won the John J. Carty Prize of the National Academy of Science. She was invited as a plenary speaker at the International Congress of Mathematicians, held in Zurich, in 1994, to become only the third woman mathematician, along with Ingrid Daubechies, to receive such an honor; Emmy Noether (in 1932) and Karen Uhlenbeck (in 1990) are the two women to have received the distinction earlier.

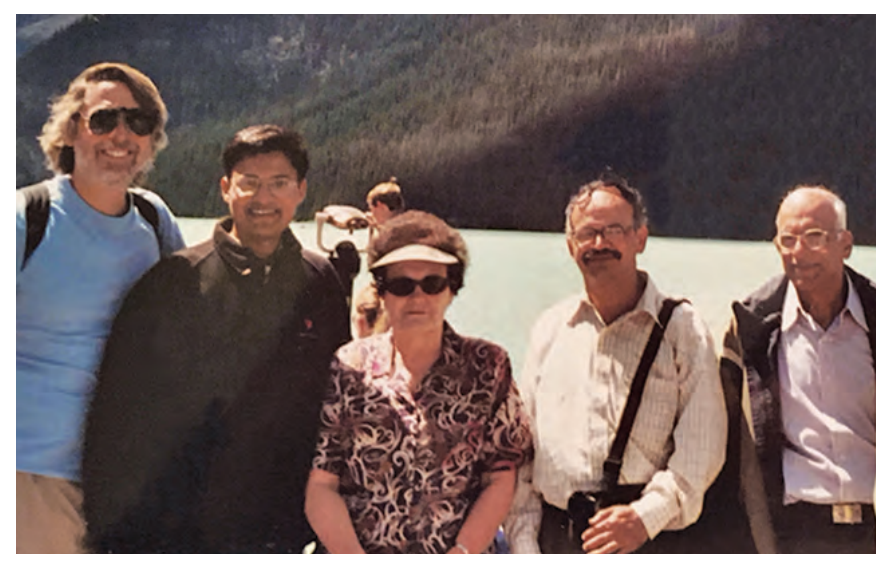

Marina Ratner at an excursion to Lake Louise, Alberta, Canada, with some of the delegates to the Conference on ErgodicTheory, held at the Banff International Research Station, Banff, Alberta, Canada, in July 2005. In the photo, from left to right, are DaveWitte Morris, Nimish Shah, Marina Ratner, S. G. Dani, and M. S. Raghunathan.

A conference on "Homogeneous Dynamics, Unipotent Flows, and Applications" was held at the Hebrew University of Jerusalem, October 13-17, 2013, in honor of Marina Ratner and her work, hosted by the Israel Institute for Advanced Studies and supported by the European Research Council. Earlier that year the Hebrew University of Jerusalem conferred upon her an honorary doctorate, at its Convocation held on June 16, 2013.

My personal contacts with Marina were, unfortunately, only sporadic, though they extended over a stretch of more than three decades. ${ }^{11}$ I found her a very warm-hearted person, going out of her way to extend hospitality, which I had numerous occasions of enjoying together with my family.

\footnotetext{
$\overline{{ }^{10} \text { The prize is awarded, since 1989, by the Ostrowski Foundation every }}$ alternate year for outstanding achievements in pure mathematics or foundations of numerical analysis.

${ }^{11}$ The first of these was in the spring of 1982 when I had an opportunity to visit the University of California, Berkeley for the semester; though in anticipation of the visit I was hoping for a serious mathematical interaction with her, it turned out, much to my disappointment, that she was on sabbatical leave during the period, which she was spending at Stanford University, and we happened to meet only occasionally during her brief visits to Berkeley.
}

We had an International Colloquium on Lie Groups and Ergodic Theory at TIFR in 1996 and the pleasure of having Ratner as one of the speakers. She also contributed a paper to the proceedings of the colloquium on $p$-adic and S-arithmetic generalizations of the Raghunathan conjecture.

My last meeting with her was in 2015, when there was a special semester organized at the Mathematical Sciences Research Institute, Berkeley, on homogeneous dynamics. On one evening she had organized a dessert party at her home. It had been a wonderful evening thanks, apart from the sumptuous desserts of wide variety, to the warm reception by Marina that she conducted so cheerfully and energetically. My wife and I got to see some photographs from her visit to Mumbai, which she had dug out for the occasion. We also got to meet her daughter Anna and her children. On receiving the news of her sad demise, I emailed Anna a condolence message expressing shock and sadness, in which I also mentioned how energetic Marina had seemed at the party. In her response Anna added, "This is all very sudden and unexpected and difficult to comprehend. She was always so full of energy." Indeed, her sad demise was very abrupt, and we deeply miss her lively presence amongst us.

\section{Credits}

Opening photo of Marina Ratner is courtesy of Anna Ratner. Photos of Marina Ratner at the 1996 International Colloquium at TIFR are courtesy of TIFR Archives.

Photo of Marina Ratner at the Hebrew University of Jerusalem, 2013 is courtesy of Israel Institute for Advanced Studies, The Hebrew University of Jerusalem.

Photo of Marina Ratner at Lake Louise is courtesy of Nimish A. Shah. 